\section{A210 COLLAGEN ANTIBODY-INDUCED ARTHRITIS (CAIA) EVOKES TRANSIENT INFLAMMATION BUT PERSISTENT ALLODYNIA}

K Sándor ${ }^{1}$, DB Bas ${ }^{1,4}$, J Petterson', J Gregory1, C Christianson ${ }^{5}$, S Codeluppi², R Holmdahl ${ }^{3}$, KS Nandakumar ${ }^{3}, \mathrm{Cl}$ Svensson ${ }^{1}$ Department of Physiology and Pharmacology, ${ }^{2}$ Department of Neuroscience, ${ }^{3}$ Department of Medical Biochemistry and Biophysics, Karolinska Institute, Stockholm, Sweden; ${ }^{4}$ Department of Pharmacology, OMU, Turkey; ${ }^{5}$ Department of Anaesthesiology, University of California, USA

\subsection{6/ard.2010.149021.20}

Background and objectives Pain is one of the most agregious symptoms reported by patients with rheumatoid arthritis (RA), a chronic disease affecting $1 \%$ of the population. RA is characterised by infiltration of inflammatory cells into the joints, synovial hypertrophy and bone erosion. Injection of CII antibodies intravenously to mice induces arthritis-like symptoms and a joint pathology that resembles human RA. While CII antibody-induced arthritis (CAIA) is a common model in the rheumatology field, it has not been evaluated as a model of arthritis-induced pain. Hence, the aim with our study was to characterise this model from a pain perspective.

Material and method $Q B$ and B10.RIII mice (males, 25-35 g), were injected with collagen antibody cocktail or saline (control) intravenously on day 0 and $25 \mu \mathrm{g}$ LPS or saline intraperitoneally on day 5. Clinical scores of arthritis (visual scoring), tactile allodynia (von Frey filaments) and locomotion (CLAMS system) were assessed for 40 days. The antinociceptive effect of diclofenac (30 mg/kg), buprenorphine $(0.1 \mathrm{mg} / \mathrm{kg})$, gabapentin $(100 \mathrm{mg} / \mathrm{kg})$, anakinra $(300 \mathrm{mg} / \mathrm{kg})$ and pentoxifylline (30 mg/kg, $30 \mu \mathrm{g}$ intrathecally) was assessed. Lumbar spinal cords were processed for immunohistochemistry (GFAP and Iba-1, marker of astrocyte and microglia activity, respectively) and quantitative PCR (GFAP, Cd11b, pituitary adenylate cyclase-activating polypeptide (PACAP) and galanin).

Results CAIA mice displayed significant increase in arthritic clinical score from days 6-18. Tactile allodynia was observed in the CAIA group throughout the study, starting prior to and outlasting reversal of the clinical score. Locomotion was significantly reduced in the postinflammatory phase in CAIA animals. Buprenorphine and gabapentin reversed CAIA-induced hypersensitivity during both the inflammatory and postinflammatory phase, while diclofenac only showed antiallodynic effect during the inflammatory phase. Surprisingly, anakinra did not display antinociceptive effect. Spinal gene and protein expression of GFAP, but not Cd11b or Iba-1, were elevated in the spinal cord in the CAIA group, as well as PACAP, a neuropeptide associated with pain modulation, both showing the most striking increase in the postinflammatory phase. Intrathecal injection of the glia-inhibitor pentoxifylline attenuated postinflammatory allodynia, pointing to potential astrocyte involvement in arthritis-associated pain.

Conclusions This study demonstrates that CAIA generates robust and highly reproducible hypersensitivity making this model suitable for studies of joint pain driven by antibody-mediated inflammation, both during peak and remittent phases of the RA. Interestingly, the tactile allodynia was prostaglandin-mediated only in the inflammatory phase, indicating that arthritis-induced pain may be driven by different mechanisms dependent on stage of disease. 